\title{
Estudio comparativo entre nifedipina-terbutalina en el trabajo de parto pretérmino
}

\author{
Rodrigo Cifuentes*; Jorge León**; Luz María De Trochez***
}

\begin{abstract}
RESUMEN OBJETIVO: Comparar la acción útero inhibidora de la Nifedipina (calcio-bloqueador) con la Terbutalina (estimulante betaadronérgico)

MATERIAL Y METODOS: Se consideraron elegibles todas las mujeres embarazadas entre la semana 26 y 35, actividad uterina aumentada, gestación única, membranas íntegras y cambios cervicales. El estudio se hizo prospectivo y randomizado, dividiendo las pacientes en dos grupos:

Uno recibió Nifedipina vía oral en dosis de $20 \mathrm{mg}$, seguido de $10 \mathrm{mg}$ cada 4 horas por 24 horas. Posteriormente $10 \mathrm{mg}$ cada 8 horas durante una semana. El grupo Terbutalina recibió $5 \mathrm{mg}$ intravenosos por 12 horas seguidos de $2.5 \mathrm{mg}$ cada 8 horas vía oral durante una semana. Hasta hoy han ingresado al estudio 46 pacientes.

RESULTADOS: Ambos fármacos han mostrado buena eficacia útero inhibidora con pocos efectos colaterales. El promedio de prolongación del embarazo desde el inicio de la terapia fue de $27=/-10$ días con Nifedipina y de $15=/-5$ días con Terbutalina ( $<<0.05$ ).

Como efecto secundario, ambos fármacos produjeron taquicardia materna y cefalea. Con Nifedipina se presentó además en la mayoría de los pacientes hipotensión arterial entre 10 y $15 \mathrm{mmHg}$.

CONCLUSION: La Nifedipina es un agente tocolítico comparable a la Terbutalina. Por causar una mayor prolongación en el embarazo, lo aconsejamos como una buena alternativa en el manejo del trabajo de parto pretérmino.

SUMMARY OBJECTIVE: To compare uterus inhibitory action of Nifedipine (blockading-calciom) with Terbutaline (beta adronergic stimulant).

MATERIAL AND METHODS: All pregnant women between 26 and 35 weeks, uterine augmented action, singular gestation, integral membranes and cervical changes were considered eligible. Study was made prospective and at random, by dividing the patients in two groups:

One of them received Nifedipine oral way in a dose of $20 \mathrm{mg}$., followed by $10 \mathrm{mg}$., every 4 hours during 24 hours. Subsequently, $10 \mathrm{mg}$, every 8 hours during a week. Terbutaline group received 5 intravenous $\mathrm{mg}$. during 12 hours followed by $2.5 \mathrm{mg}$. each 8 hours oral way during a week. Till today, 46 patients have become a part of the study.

RESULTS: Both medicines have showed a good uterus inhibitory efficacy with few collateral effects. The standar of pregnancy lengthening from the begining of the therapy was of $27=/-10$ days with Nifedipine and of $15=/$ - days with Terbutaline (p 0.05 ).

As a secondary effect, both medicines produced maternal tachycardia and violent headache. Arterial hypotension between 10 and 15 $\mathrm{mmHg}$, was presented, moreover, with Nifedipine, in a large number of patients.

CONCLUSION: Nifedipine is a tocolitic agent comparable with Terbutaline. As it causes a longer prolongation of pregnancy, we suggest it as a good alternative in preterm childbirth labor management.
\end{abstract}

\section{Introducción}

La prematuridad y el bajo peso al nacer (BPN) continúan siendo un problema de salud pública.

La incidencia del BPN en el Hospital Universitario del Valle en una muestra poblacional realizada en 1989 fue del $12,9 \%$ (1); porcentaje considerablemente mayor comparada a la incidencia presentada en estudios publicados en los Estados Unidos, los cuales se mantienen alrededor del $8 \%$ de todos los nacidos vivos (2).

La Tocolisis, es la inhibición farmacológica de las contracciones uterinas. Es la principal medida preventiva del Trabajo de Parto Pretérmino (TPP) y se mantiene hasta tanto la etiología de dicha patología sea aclarada.

Profesor Titular. Jefe Sección Medicina Perinatal. Facultad de Salud. Universidad del Valle, Cali.

** Residente Universidad de Los Andes. San Cristóbal, Venezuela. Rotante por Sección de Medicina Perinatal, Universidad del Valle.

*** Laboratorio de Medicina Perinatal.
Aunque los agentes Beta-simpaticomiméticos presentan un rápido efecto úteroinhibidor cuando se administran por vía endovenosa (EV), éstos tienen el inconveniente de que inducen en el binomio madre-feto, una serie de efectos colaterales que pueden ser severos; Ejemplo: Edema pulmonar, isquemia cardíaca y cardiotoxicidad fetal $(3,4)$; o menos serios pero más prevalentes como son la taquicardia, ansiedad, palpitaciones o insomnio, (5); efectos que se traducen en intolerancia materna y conllevan a disminuir la dosis o a suspender el fármaco presentándose en muchos casos fracasos en el tratamiento.

Desde 1978 se han publicado varios trabajos sobre la eficacia de los calcio-antagonistas (Nifedipina) en disminuir o prevenir la contracción del músculo liso uterino (68).

La nifedipina, un agente bloqueador de los canales del calcio, ha emergido como un agente tocolítico potencialmente seguro y mejor tolerado que otros úteroinhibidores. Este fármaco inhibe la contracción del músculo liso impidiendo el flujo del calcio a través de la membrana celular 
(8). Los efectos colaterales maternos son mínimos consistiendo en cefalea transitoria y rubor $(6,9)$.

Aunque la seguridad de la nifedipina ha sido cuestionada según estudios realizados en animales, por producir disminución de la pO2 arterial fetal y el pH (10-11) no hay datos reportados sobre efectos fetales adversos en humanos.

Hay estudios que demuestran la eficacia de la nifedipina como úteroinhibidor, pero existen pocos estudios comparativos del mismo con otros fármacos úteroinhibidores.

El objetivo de este estudio es comparar la acción úteroinhibidora de la nifedipina: Bloqueador de los canales del calcio; con los de la terbutalina: Estimulante de los receptores Beta-adrenérgicos. Se desea igualmente cuantificar los efectos colaterales de ambos fármacos sobre el binomio madre-feto.

\section{Pacientes y métodos}

Se estudiaron prospectivamente 46 pacientes embarazadas entre la semana 26 a la 35 , ingresadas al Hospital Universitario del Valle desde el 01 de julio al 30 de noviembre de 1993.

Dichas pacientes debían presentar al menos una contracción uterina en 10 minutos con duración mínima_de $30 \mathrm{seg}$. gestación única, membranas ovulares íntegras y con cambios cervicales de borramiento y/o dilatación.

El diagnóstico de edad gestacional se basó en la determinación precisa de la fecha de la última menstruación (FUM), corroborada por series ecográficas y por controles clínicos en la consulta de Embarazo de Alto Riesgo (EAR).

La contractilidad uterina (CU) se detectó clínicamente y se confirmó con registro externo electrónico (monitor fetal corometrics No. 116).

Se realizó valoración cervical incluyendo en el estudio sólo pacientes con borramiento de hasta el $50 \%$ y dilatación máxima de $4 \mathrm{cms}$.

Las pacientes elegibles para el estudio se les descartó al ingreso de patologías cardiovasculares, trastornos hipertensivos, RPM, RCIU, diabetes y problemas infecciosos de tipo viral.

Se realizó monitoría fetal electrónica de la Frecuencia Cardíaca Fetal (FCF) y registro externo de la CU a las 46 pacientes durante 30 minutos al cabo de los cuales se administró el tratamiento úteroinhibidor.

De manera aleatorizada a 23 pacientes se les administró nifedipina: $20 \mathrm{mg}$ VO inicial, seguidas de $10 \mathrm{mg}$ VO cada 4 horas por 24 horas y posteriormente se les continuó con 10 mg VO cada 8 horas por 7 días.

En el otro grupo a las pacientes se les administró terbutalina: ( $5 \mathrm{mcg} / \mathrm{minut}) \mathrm{EV}$ en 12 horas, seguidos de 2,5 mg VO cada 8 horas por 7 días. A todas las embarazadas se les continuó el registro de la CU y de la FCF hasta al menos 1 hora de administrado el fármaco úteroinhibidor.

En el registro de la monitoría se valoró la frecuencia y duración de las contracciones uterinas, y se calculó "actividad uterina" reemplazando la intensidad de la contracción por su duración.

La presión arterial y el pulso se valoraron previo a la administración de los fármacos y a los 15 y 30 minutos respectivamente luego de la dosis inicial.
Se les realizó a todas las pacientes historia clínica perinatal, se solicitaron exámenes de laboratorio (cuadro hemático, parcial de orina, VDRL, frotis cervical), y se admitieron a la unidad de cuidados especiales del servicio de medicina perinatal del Hospital Universitario del Valle.

Se realizó Perfil Biofísico Fetal (PBF) antes del ingreso al estudio y 72 horas después de iniciada la terapia.

Las pacientes permanecieron hospitalizadas por lo menos durante 72 horas tiempo durante el cual recibieron también betametasona en dosis de $12 \mathrm{mg}$. iniciales y $12 \mathrm{mg}$. 24 horas después para inducir maduración pulmonar.

Las pacientes egresaban 12 horas después de no presentar contractilidad uterina y se controlaron semanalmente por la consulta de EAR, atendiéndose los partos de las mismas en este centro hospitalario.

\section{Resultados}

La edad materna en ambos grupos de estudio fue de 23 años en promedio (Tabla 1).

El promedio de edad gestacional y paridad fue también similar en ambos grupos, no presentándose diferencias estadísticamente significativas (Tabla 1).

\section{Tabla 1 \\ NIFEDIPINA-TERBUTALINA FACTORES MATERNOS}

\begin{tabular}{|cccc|}
\hline Clasificación & Nifedipina & Terbutalina & $\mathbf{P}<\mathbf{0 . 0 5}$ \\
\hline Edad materna* $^{*}$ & $23.0 \pm 4.9$ & $22.5 \pm 5.9$ & $\mathrm{NS}$ \\
E. Gestacional & $32.8 \pm 2.4$ & $32.5 \pm 2.6$ & $\mathrm{NS}$ \\
Paridad: 0 $^{*}$ & $5 / 23$ & $4 / 23$ & $\mathrm{NS}$ \\
mayor 0 & $18 / 23$ & $19 / 23$ & $\mathrm{NS}$ \\
\hline
\end{tabular}

* Media \pm DS. Análisis t student.

Se observa que la edad materna, edad gestacional y la paridad fueron similares en ambos grupos de estudio.

La duración de la contracción, la frecuencia y la "Actividad uterina" fueron también similares en ambos grupos, previo al tratamiento úteroinhibidor.

Posterior al tratamiento con ambos fármacos, se demuestra estadísticamente $(\mathrm{p}<0.05)$ que tanto la nifedipina como la terbutalina son excelentes úteroinhibidores. (Tabla 2, Figs. 1-4). Cuando se estudiaron los parámetros duración de la contracción uterina, frecuencia de las contracciones y "Actividad Uterina" nos encontramos que el parámetro que más se afectaba era la duración de la contracción seguida de la actividad con resultados estadísticamente significativos. (Tabla 2, Figs. 3 y 4 ).

En cuanto a parámetros de presión arterial y pulso materno, lo resaltante fue el aumento de la frecuencia cardíaca materna a los 30 minutos de la dosis inicial de terbutalina; aumento estadísticamente significativo comparado con la nifedipina, (Tabla 3 ).

Cuando se estudió prolongación de la gestación se encontró que las pacientes que recibieron nifedipina se les prolongó el embarazo aproximadamente el doble del tiempo de aquellas pacientes que recibieron terbutalina. $(\mathrm{p}<0.05)$ Figura 5. 
Tabla 2

NIFEDIPINA-TERBUTALINA. DURACION DE LA CONTRACCION, FRECUENCIA Y "ACTIVIDAD UTERINA" PRE Y POST TRATAMIENTO

\begin{tabular}{|c|c|c|c|c|c|c|}
\hline \multirow{2}{*}{ Clasificación } & \multicolumn{3}{|c|}{ Nifedipina } & \multicolumn{3}{|c|}{ Terbutalina } \\
\hline & Pre & Post & $\mathrm{P}<0.05$ & Pre & Post & $P<0.05$ \\
\hline Duración $*^{\circ}$ & $51.1 \pm 15$ & $25.5 \pm 19.5$ & S & $50 \pm 15$ & $34.9 \pm 13$ & S \\
\hline Frecuencia* & $4.2 \pm 0.7$ & $1.9 \pm 1.5$ & NS & $3.9 \pm 1$ & $2.4 \pm 1.1$ & NS \\
\hline "Act. Uterina"* & $217.8 \pm 75$ & $71.4 \pm 55$ & $\mathrm{~S}$ & $197.6 \pm 70$ & $84.7 \pm 64$ & $\mathrm{~S}$ \\
\hline
\end{tabular}

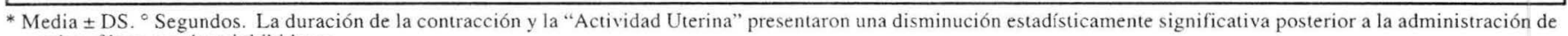
ambos fármacos úteroinhibidores.

Figura 1

NIFEDIPINA-TERBUTALINA. REGISTRO EXTERNO DE LA CONTRACCION UTERINA PRE Y POST ADMINISTRACION DE NIFEDIPINA
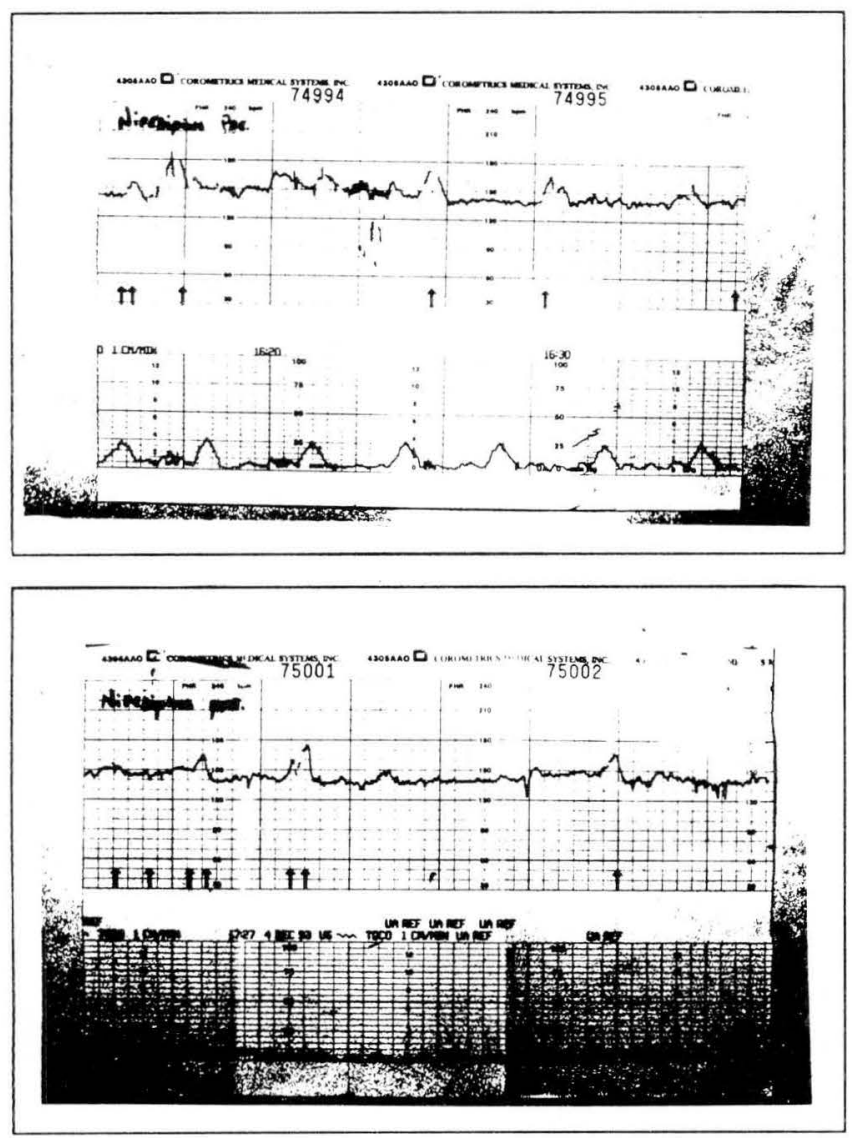

Figura 2

NIFEDIPINA-TERBUTALINA. REGISTRO

EXTERNO DE LA CONTRACCION UTERINA PRE Y POST ADMINISTRACION DE TERBUTALINA

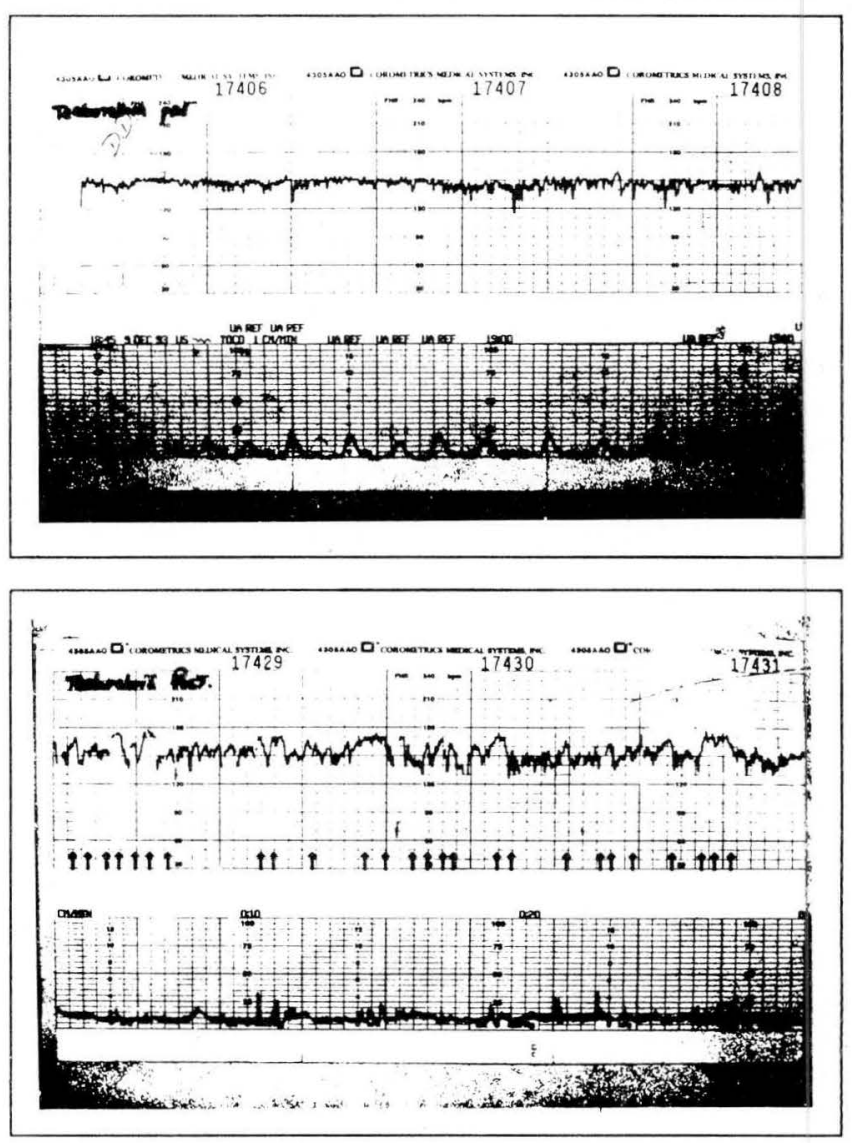

Tanto en la figura 1 como en la 2 se aprecia la eficacia úteroinhibidora tanto de la nifedipina sumo de la terbutalina.

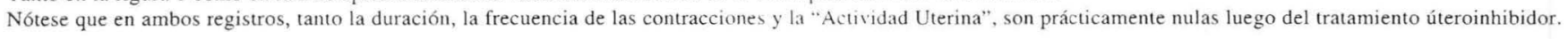

En cuanto al peso de los recién nacidos, se aprecia que los neonatos producto de las pacientes que recibieron nifedipina presentaron un peso estadísticamente mayor que el peso de aquellos cuyas madres recibieron terbutalina. $(p<0.05)$ Figura 6.

El efecto colateral más frecuente en las pacientes que recibieron nifedipina fue la cefalea (30.4\%); esta cefalea era de leve intensidad y cedía con la administración de analgésicos comunes. (Acetaminofén).
Con respecto a las pacientes que recibieron terbutalina, los efectos colaterales más frecuentes fueron las palpitaciones $(43.4 \%)$ seguidos de disnea $(30.4 \%)$ (Tabla 4$)$.

\section{Discusión}

Al comparar la acción tocolítica de la nifedipina (calcioantagonista) con la terbutalina (estimulante Betaadrenérgico) en pacientes con TPP en nuestro centro, hemos 
Figura 3

NIFEDIPINA-TERBUTALINA. DURACION

DE LAS CONTRACCIONES UTERINAS ANTES

Y DESPUES DEL TRATAMIENTO

UTEROINHIBIDOR

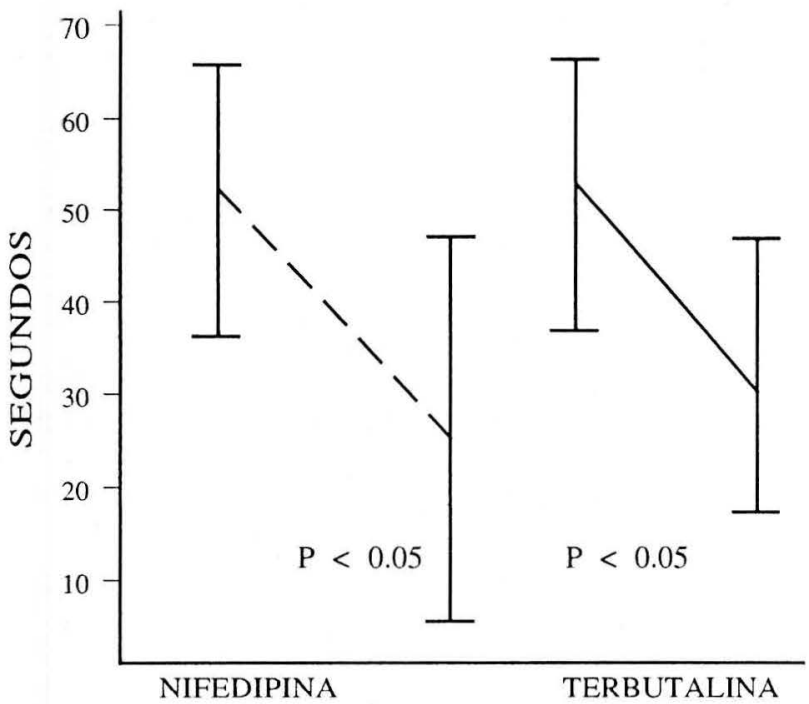

Se aprecia disminución estadísticamente significativa de la duración de las contracciones uterinas posterior a la administración de ambos fármacos úteroinhibidores.

Figura 4
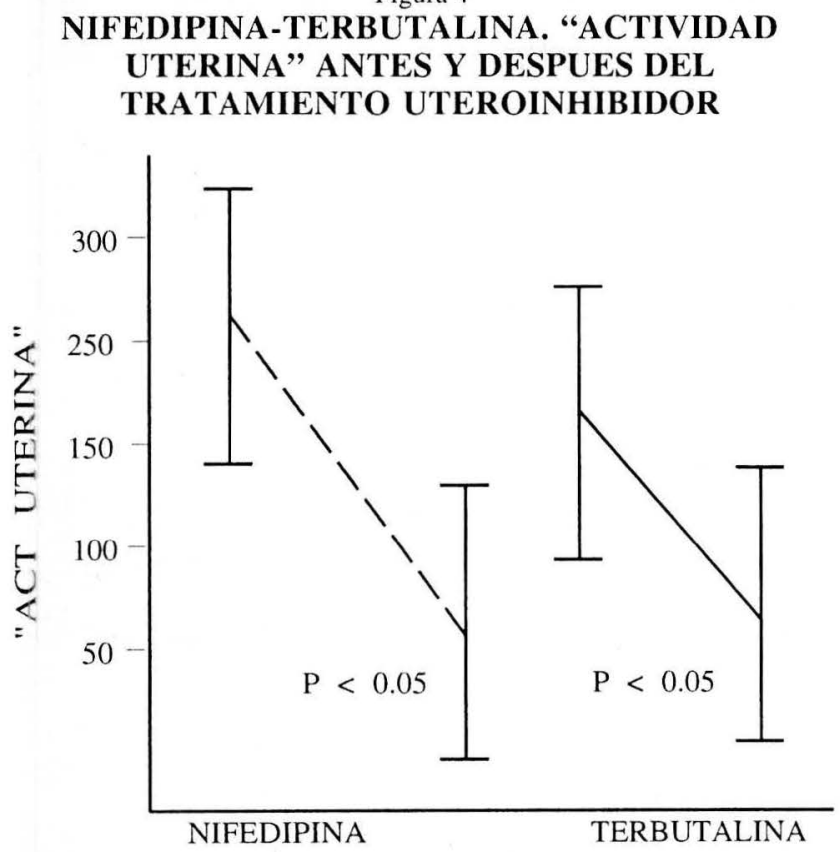

Obsérvese la disminución de la "Actividad uterina" posterior al tratamiento úteroinhibidor.

podido constatar y equiparar resultados estadísticamente significativos y comparables a los obtenidos por otros autores a nivel mundial $(7,12)$.

Se demuestra la eficaz acción tocolítica tanto de la nifedipina $(\mathrm{p}<0.05)$ como de la terbutalina $(\mathrm{p}<0.05)$, acción

\section{Figura 5 \\ NIFEDIPINA-TERBUTALINA. PROLONGACION DEL EMBARAZO (EN DIAS) EN EL TRABAJO DE PARTO PRETERMINO}

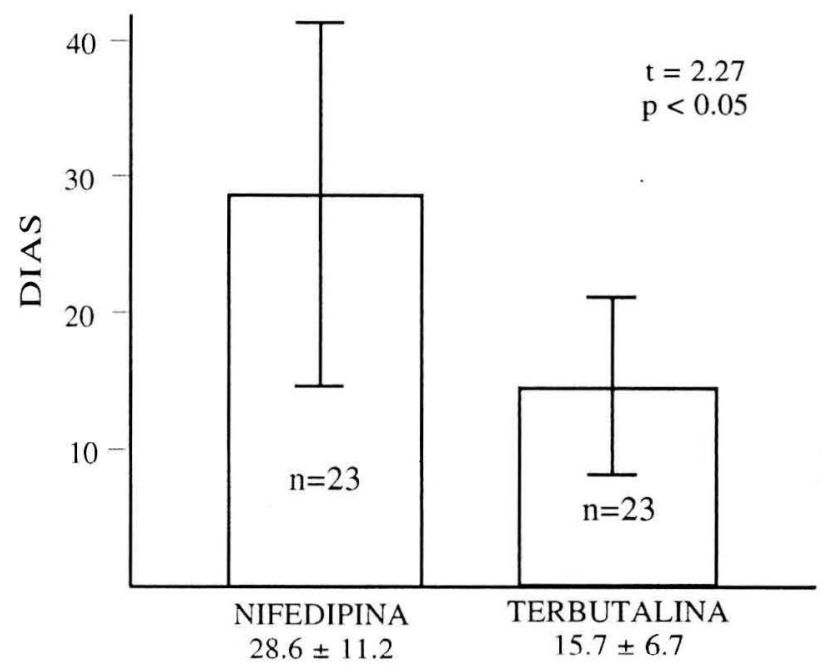

Se presentó mayor prolongación del tiempo de embarazo en las pacientes que recibieron nifedipina como úteroinhibidor.

\section{Figura 6 \\ NIFEDIPINA-TERBUTALINA. PESO DE LOS RECIEN NACIDOS}

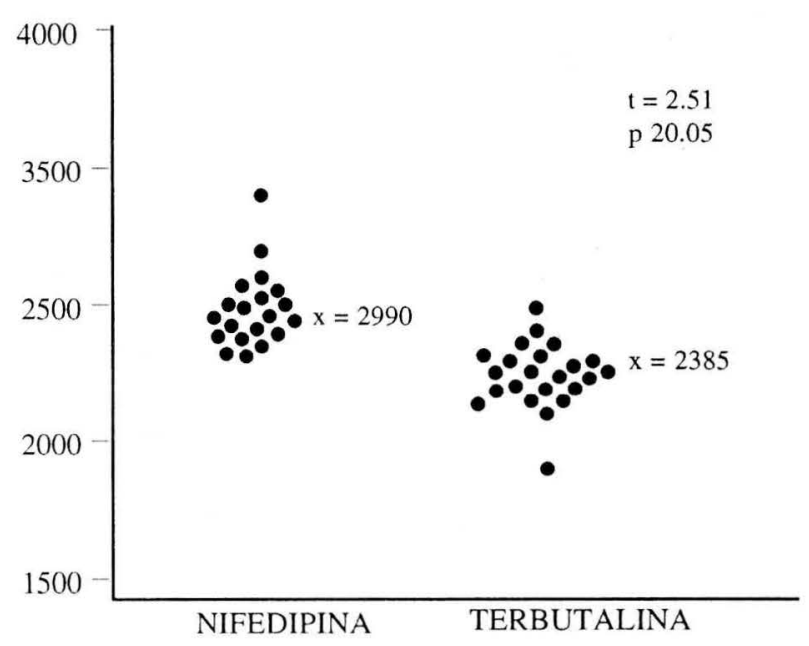

Los recién nacidos hijos de pacientes que recibieron nifedipina presentaron un peso promedio al nacer mayor que los $\mathrm{RN}$ hijos de madres del grupo terbutalina; esto probablemente debido a la mayor prolongación del embarazo en el grupo de nifedipina.

inicial y predominantemente dirigida hacia la duración de la contracción uterina.

En nuestro estudio, realizado en forma prospectiva y aleatoria obtuvimos una mayor prolongación de la gestación $(\mathrm{p}<0.05)$ y mayor promedio de peso de $\operatorname{los} \mathrm{RN}(\mathrm{p}<0.05)$ en las pacientes que recibieron nifedipina. 
Tabla 3

NIFEDIPINA-TERBUTALINA. PRESION ARTERIAL Y FRECUENCIA CARDIACA MATERNA PRE Y POST TRATAMIENTO

\begin{tabular}{|c|c|c|c|c|c|c|c|c|}
\hline \multirow[t]{2}{*}{ Clasificación } & \multicolumn{3}{|c|}{ Nifedipina } & \multirow{2}{*}{$\begin{array}{l}\mathscr{n} \\
\stackrel{\vec{v}}{v} \\
\tilde{v}\end{array}$} & \multicolumn{3}{|c|}{ Terbutalina } & \multirow{2}{*}{$\begin{array}{l}\text { ro } \\
\stackrel{\dot{\hat{v}}}{\mathrm{v}} \\
\text { a. }\end{array}$} \\
\hline & Inicial & 15 Min & 30 Min & & Inicial & 15 Min & 30 Min & \\
\hline P. Arterial* & $113 / 68.0$ & $108.9 / 62.3$ & $108.7 / 60.4$ & NS & $112.6 / 68.9$ & $108.0 / 66.3$ & $107 / 64.3$ & NS \\
\hline F. Card. Mat ${ }^{\circ}$ : & $82.8 \pm 6.7$ & $89.4 \pm 5.7$ & $92.8 \pm 6.0$ & NS & $83.7 \pm 4.9$ & $98.4 \pm 5.1$ & $107.8 \pm 6$ & $S$ \\
\hline
\end{tabular}

* Promedio +Media $\pm{ }^{\circ} \mathrm{DS}$. Análisis t student.

Lo estadísticamente significativo es el aumento de la frecuencia cardíaca a los 30 minutos luego de la administración EV de terbutalina $(\mathrm{p}<0.05)$.

Una característica importante de las pacientes que recibieron nifedipina, es que a pesar de la cefalea que presentaron algunas, la mayoría refería una mejoría sintomática al poco tiempo de iniciada la terapia y aún existiendo contractilidad uterina considerable.

Datos también importantes son los referidos a la mínima presencia de efectos colaterales en las pacientes que fueron tratadas con nifedipina en comparación con las del grupo terbutalina.

Los datos anteriores y los resultados altamente satisfactorios obtenidos con la nifedipina nos permite concluir que este fármaco es un excelente y potente tocolítico comparable a otros más frecuentemente usados.

El bajo costo de la nifedipina, su comprobada eficacia, su fácil y cómoda administración, su poca frecuencia de efectos colaterales nos permite aconsejarlo como una excelente opción terapéutica en el manejo del Trabajo de Parto Pretérmino.

\section{Agradecimientos}

Nuestro agradecimiento al Dr. Hugo Hurtado, Magister en Salud Pública de la Universidad del Valle, por su valiosa colaboración en el proceso de datos y asesoría estadística.
Tabla 4

\section{NIFEDIPINA-TERBUTALINA. EFECTOS COLATERALES}

\begin{tabular}{|lccrc|}
\hline Clasificación & \multicolumn{2}{c}{ Nifedipina } & \multicolumn{2}{c|}{ Terbutalina } \\
& No. & \% & No. & \% \\
\hline Cefalea & 7 & 30.4 & 2 & 8.6 \\
Rubor & 3 & 13.0 & 0 & 0 \\
Palpitaciones & 0 & 0 & 10 & 43.4 \\
Náuseas y Vóm. 2 & 8.6 & 1 & 4.3 \\
Disnea & 0 & 0 & 7 & 30.4 \\
Taquicard. 120 & 0 & 0 & 3 & 13.0 \\
\hline
\end{tabular}

El efecto colateral más frecuente en las pacientes tratadas con nifedipina fue la cefalea, en tanto que el efecto colateral más frecuente en las pacientes del grupo terbutalina fueron las palpitaciones seguidas de disnea.

\section{BIBLIOGRAFIA}

1. Cifuentes R. parto Pretérmino. II Congreso Nacional Científico y Gremial y X Congreso de Medicina General y Social. Cali. Col. Nov. 1989.

2. American College of Obstetricians and Gynecologists. Preterm Labor. ACOG. Tech Bull. 1989; 133.

3. Katz V., Seeds J. Fetal and Neonatal Cardiovascular complications from Beta-simpathomimetics therapy for tocolysis. Am. J. Obstet. Gynecol. 1989; 161: 1-4.

4. Benedetti TJ. Maternal complications of parenteral B-simpathomimetics therapy for premature labor. Am. J. Obstet. Gynecol. 1983; 145: 1-6.

5. Physician' desk reference. 43rd, Oradell, New Jersey: Medical Economics, 1989; 649-650.

6. Ulstem U. "Relaxing effects on nifedipine on the no pregnant human uterus in vivo and in vitro"; Obst.Gynecol. Vol. 52. oct. 1978; 411436.

7. Csapo A et al. "Deactivacion of the uterus during normal and premature labor by the calcium antagonist nicardipine". Am. J. Obstet. Gynecol. 142.5. Marzo 1982.
8. Forman A., Anderson K., Wingerup L. Treatment of the premature labor with the calcium antagonist nifedipine. Arch Gynecol 229.1.1981.

9. Kaul A., Osathonondh F., Safon L., Frigoletto F. The management of preterm labor with the calcium channel blocking agent nifedipine combined with terbutaline. Drug Intell Clin Pharm. 1985; 19: 369371.

10. Ducsay CA., Thompson JS., Wu AT., Novy MJ. Effects of calcium blocker (nicardipine) tocolisis in rhesus macaques: Fetal plasma concentrations and cardiorespiratory changes. Am. J. Obstet. Gynecol. 1987; 157: 1482-1486.

11. Harake B., Gilbert R., Ashwal S., Power G. Nifedipine: Effects on fetal and maternal hemodynamics in pregnant sheep Am. J. Obstet. Gynecol. 1987; 157: 1482-1486.

12. Read M., Wellby D. The use of a calcium antagonist (nifedipine) to supress preterm labor. Br. J. Obstet. Gynecol. 1986; 93: 933-937. 